\title{
The inner two degrees of the Milky Way
}

\section{Evidence of a chemical difference between the Galactic Center and the surrounding inner bulge stellar populations}

\author{
M. Schultheis ${ }^{1}$, R. M. Rich ${ }^{2}$, L. Origlia ${ }^{3}$, N. Ryde ${ }^{4}$, G. Nandakumar $^{5,6}$, B. Thorsbro ${ }^{4}$, and N. Neumayer ${ }^{7}$ \\ 1 Université Côte d'Azur, Observatoire de la Côte d'Azur, CNRS, Laboratoire Lagrange, Blvd de l'Observatoire, 06304 Nice, France \\ e-mail: mathias.schultheis@oca.eu \\ 2 Department of Physics and Astronomy, UCLA, 430 Portola Plaza, Box 951547 Los Angeles, CA 90095-1547, USA \\ e-mail: rmr@astro.ucla.edu \\ 3 INAF-Osservatorio di Astrofisica e Scienza dello Spazio, Via Gobetti 93/3, 40129 Bologna, Italy \\ ${ }^{4}$ Lund Observatory, Department of Astronomy and Theoretical Physics, Lund University, Box 43, 22100 Lund, Sweden \\ 5 Research School of Astronomy \& Astrophysics, Australian National University, ACT 2611, Australia \\ 6 ARC Centre of Excellence for All Sky Astrophysics in Three Dimensions (ASTRO-3D), Australia \\ 7 Max-Planck-Institut für Astronomie, Königstuhl 17, 69117 Heidelberg, Germany
}

Received 25 April 2019 / Accepted 18 June 2019

\begin{abstract}
Context. Although there have been numerous studies of chemical abundances in the Galactic bulge, the central two degrees have been relatively unexplored due to the heavy and variable interstellar extinction, extreme stellar crowding, and the presence of complex foreground disk stellar populations.

Aims. In this paper we discuss the metallicity distribution function, vertical and radial gradients, and chemical abundances of $\alpha$-elements in the inner two degrees of the Milky Way, as obtained by recent IR spectroscopic surveys.

Methods. We used a compilation of recent measurements of metallicities and $\alpha$-element abundances derived from medium-high resolution spectroscopy. We compare these metallicities with low-resolution studies.

Results. Defining "metal-rich" as stars with $[\mathrm{Fe} / \mathrm{H}]>0$, and "metal-poor" as stars with $[\mathrm{Fe} / \mathrm{H}]<0$, we find compelling evidence for a higher fraction ( $\sim 80 \%)$ of metal-rich stars in the Galactic Center (GC) compared to the values (50-60\%) measured in the low latitude fields within the innermost $600 \mathrm{pc}$. The high fraction of metal-rich stars in the GC region implies a very high mean metallicity of +0.2 dex, while in the inner $600 \mathrm{pc}$ of the bulge the mean metallicity is rather homogenous around the solar value. A vertical metallicity gradient of $-0.27 \mathrm{dex} \mathrm{kpc}^{-1}$ in the inner $600 \mathrm{pc}$ is only measured if the GC is included, otherwise the distribution is about flat and consistent with no vertical gradient.

Conclusions. In addition to its high stellar density, the Galactic center/nuclear star cluster is also extreme in hosting high stellar abundances, compared to the surrounding inner bulge stellar populations; this has implications for formation scenarios and strengthens the case for the nuclear star cluster being a distinct stellar system.
\end{abstract}

Key words. Galaxy: bulge - Galaxy: center - Galaxy: stellar content - stars: fundamental parameters - stars: abundances infrared: stars

\section{Introduction}

The era of large spectroscopic surveys has strengthened the case that the central regions of the galaxy are dominated by an old metal-rich bulge/bar stellar population that is distinct in formation history from the thin disk (see e.g., the reviews of Rich 2013; Origlia 2014, and Barbuy et al. 2018). The vast majority of these studies have been done at optical wavelengths $(<1 \mu \mathrm{m})$ and in regions of relatively low interstellar extinction at $b<-2^{\circ}$, such as the well-known Baade's window (BW), although recent efforts have probed the bulge closer to the plane (Zoccali et al. 2017; Trapp et al. 2018). In these regions, due to the largeand high-variable interstellar extinction (see e.g., Gonzalez et al. 2012; Schultheis et al. 2014; Nogueras-Lara et al. 2018a), observations are limited to the near-infrared and longer wavelengths. With the recent development of high-resolution IR spectrographs, it is now possible to get detailed chemical abundances of giant stars in the inner Galactic bulge, although cool giants (with substantial molecular lines) remain a challenge for abundance analysis. We define here the inner Galactic bulge with $|l|<10^{\circ},|b| \leq 3^{\circ}$ assuming a distance to the Galactic Center (GC) of $8.2 \mathrm{kpc}$ (Karim \& Mamajek 2017) ${ }^{1}$. Early high-resolution IR spectroscopy commenced with the Near Infrared Spectrometer (NIRSPEC) on Keck II (Rich et al. 2007, 2012), and then on larger samples with the Apache Point Observatory Galaxy Evolution Experiment (APOGEE) survey (Majewski et al. 2017). As was the case in the earlier studies with NIRSPEC, APOGEE works in the $H$-band where extinction can still be significant $\left(A_{\mathrm{V}} / A_{H} \sim 6\right)$, especially in the inner degree where it reaches extreme values (see e.g., Schultheis et al. 2009; Fritz et al. 2011; Nogueras-Lara et al. 2018a). $K$-band spectroscopy $\left(A_{\mathrm{V}} / A_{K} \sim 10\right)$ of $\mathrm{M}$ giants is thus the most efficient way to measure detailed chemical signatures in the innermost regions (see e.g., Cunha et al. 2007; Ryde \& Schultheis 2015; Nandakumar et al. 2018). Figure 1 shows a view of the different

\footnotetext{
1 degree corresponds to $145 \mathrm{pc}$.
} 


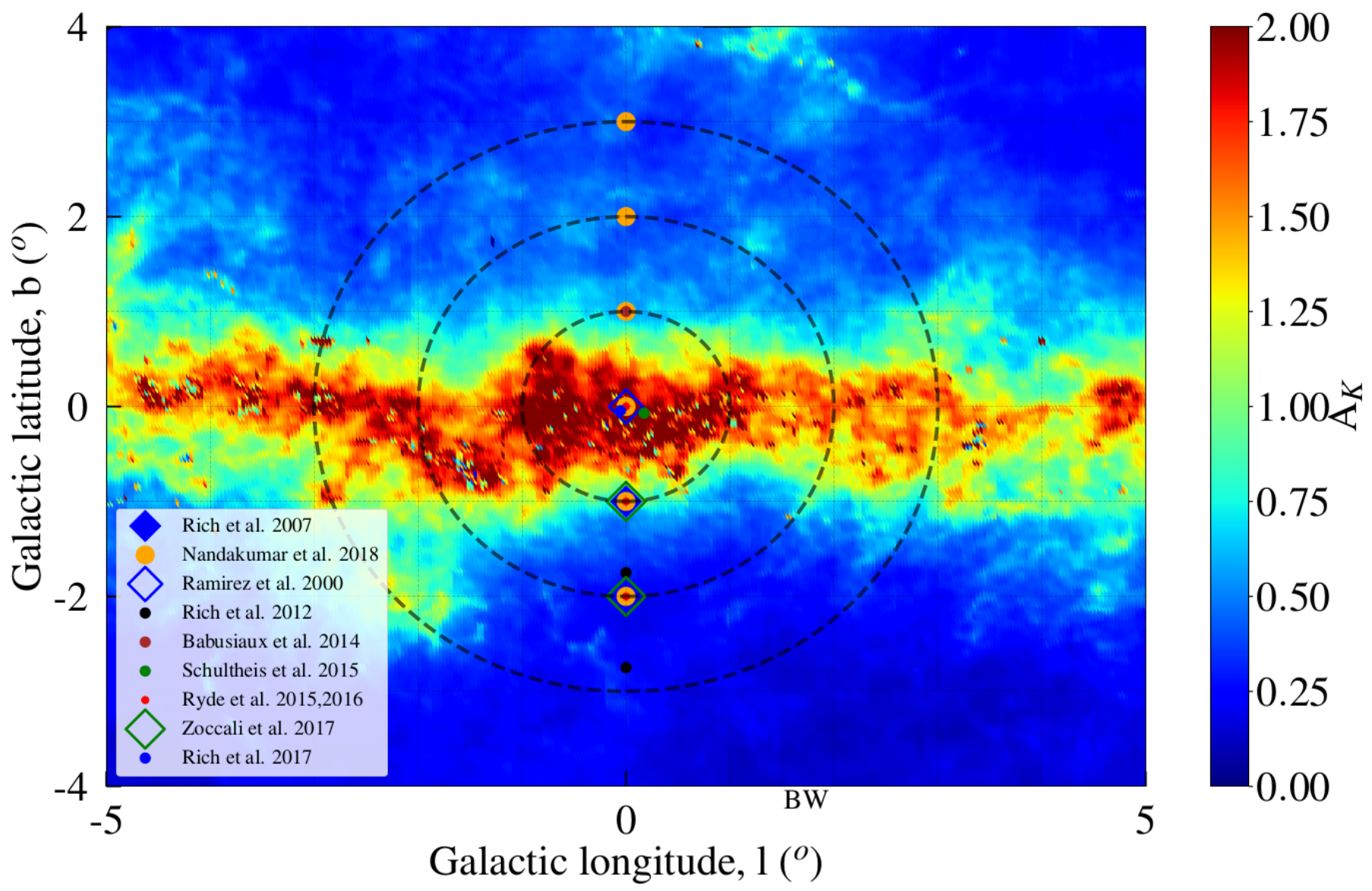

Fig. 1. Location of target fields from spectroscopic studies in inner bulge. The data is superimposed on the interstellar extinction map of Gonzalez et al. (2012). The dashed lines denote an angular radius of 1,2, and 3 degrees from the Galactic Center, respectively.

studies obtained in the inner Galactic bulge superimposed to the extinction map of Gonzalez et al. (2012).

In the central $5 \mathrm{pc}$, the stellar population is dominated by the nuclear star cluster, which hosts a wide range of stellar ages and metallicities, even including young massive stars (Genzel et al. 2010). Nuclear star clusters (NSCs) are in general the most dense stellar systems in the universe, with masses of $10^{7} M_{\odot}$ and typical sizes of $\sim 5 \mathrm{pc}$ (see Neumayer 2017). The NSC of the Milky Way has an estimated half-light radius of $4.2 \pm 0.4 \mathrm{pc}$ (Schödel et al. 2014) and a mass of $2 \times 10^{7} M_{\odot}$ (Fritz et al. 2016; Feldmeier et al. 2014), and it also hosts the supermassive black hole, $\mathrm{SgrA}^{*}$. Its formation mechanism is still under debate. Two main scenarios have been proposed: (i) stars have formed in situ in the center of the Milky Way (e.g., Seth et al. 2008; Milosavljević 2004; Pflamm-Altenburg \& Kroupa 2009); and (ii) star clusters have migrated from a larger distance (e.g., Tremaine et al. 1975; Capuzzo-Dolcetta 1993; Antonini 2013, Gnedin et al. 2014). The metallicity distribution function, as well as detailed chemical abundances, are important constraints on the formation history of the NSC and inner bulge close to the Galactic plane. In the NSC, the cool stars amenable to abundance determination can run the gamut from red supergiants several Myr old, to red giants $t \geq 10$ Gyr old. Carr et al. (2000) and Ramírez et al. (2000a) carried out detailed abundance analysis using high-resolution IR spectra $(R \sim 40000)$ and estimated a mean metallicity of $+0.12 \pm 0.22$ dex for ten cool luminous supergiant stars in the GC. The same stars were remeasured by Cunha et al. (2007) with a slightly higher resolution $(R \sim$ $50000)$ and they estimated a similar mean metallicity. However, red supergiant stars have complex stellar atmospheres that are challenging to model and the analysis of such spectra is therefore nontrivial (see e.g., Cunha \& Smith 2006). Ramírez et al. (2000a) determined, for the first time, metallicities of $\mathbf{M}$ giant stars in bulge fields along the minor axis using low-resolution $(R \sim 1300-4800)$ spectra. They determined metallicities based on the equivalent widths of three strong features in their $K$-band spectra, namely NaI, CaI, and the first overtone band of $\mathrm{CO}$. They did not find any evidence for a metallicity gradient along the minor or major axes in the inner bulge $\left(R_{\mathrm{GC}} \lesssim 560 \mathrm{pc}\right)$.

Rich et al. (2007) did one of the first detailed abundance determination from high-resolution IR spectroscopy with NIRSPEC (McLean 2005) at Keck II, by analysing $17 \mathrm{M}$ giants located at $(l, b)=\left(0^{\circ},-1^{\circ}\right)$. They found a mean iron abundance of $[\mathrm{Fe} / \mathrm{H}]=-0.22$ with a $1 \sigma$ dispersion of 0.14 dex. Later on, Rich et al. (2012) carried out a consistent analysis of $30 \mathrm{M}$ giants at $(l, b)=(0,-1.75)$ and $\left(1^{\circ},-2.75\right)$ that were observed with the same instrument and estimated mean iron abundances of $-0.16 \pm 0.12 \mathrm{dex}$ and $-0.21 \pm 0.08 \mathrm{dex}$, respectively. These authors also combined their analysis of $14 \mathrm{M}$ giants in BW $(l=$ $1.02, b=-3.93)$ using the same instrument and Rich \& Origlia (2005) found no gradient in abundance nor $[\alpha / \mathrm{Fe}]$ in the innermost $150 \mathrm{pc}$ to $600 \mathrm{pc}$ region. Babusiaux et al. (2014) determined metallicities for $\sim 100$ red clump $(\mathrm{RC})$ stars at $(l, b)=$ $\left(0^{\circ},+1^{\circ}\right)$ using low-resolution optical spectra $(R \sim 6500)$ and found a mean metallicity very similar to that of Ramírez et al. (2000b) and Rich et al. (2007) at $(l, b)=\left(0^{\circ},-1^{\circ}\right)$, suggesting symmetry between northern and southern inner bulge fields. 
Schultheis et al. (2015) analysed APOGEE spectra of cool $\mathrm{M}$ giants in the inner degree, finding a significant presence of a metal-poor population enhanced in $\alpha$-elements. However, interstellar extinction limited APOGEE observations near the GC, and only some red supergiants and luminous asymptotic giant branch stars (see Schultheis et al. 2015) could be measured.

Do et al. (2015) derived metallicities for about $80 \mathrm{M}$ giants in the NSC observed at low resolution $(R \simeq 5000)$ with GeminiNorth's Near-Infrared Integral Field Spectrometer (NIFS). Their Bayesian analysis reported a wide range in derived metallicities, extending to +0.96 dex, and with $6 \%$ of their sample having $[\mathrm{Fe} / \mathrm{H}]<-0.5$. However, their abundances can be considered to be only estimates, as they also reported $\log g>3$ for most of their sample.

Feldmeier-Krause et al. (2017) used a method similar to Do et al. (2015), reporting approximate metallicities for 700 $M$ giants in the vicintiy of the NSC, using VLT-KMOS spectroscopy at $R=4000$. They also reported a suprasolar metallicity and a metal poor fraction similar to that of Do et al. (2015). Nogueras-Lara et al. (2018b) used a purely photometric result and fitted luminosity functions to their deep photometric data in the inner bulge with $\alpha$-enhanced BASTI isochrones and found that an old stellar population with $Z=0.04$ giving a $[\mathrm{Fe} / \mathrm{H}]=0.18$ dex provides the best fit.

One of the first detailed abundance analyses in the GC using $K$-band, high-resolution the CRyogenic high-resolution InfraRed Echelle Spectrograph (CRIRES) at the ESO Very Large Telescope (VLT) spectra were obtained by Ryde \& Schultheis (2015). They analyzed spectra of nine field giants in the vicinity of the NSC, finding a narrow metallicity distribution with $[\mathrm{Fe} / \mathrm{H}]=+0.11 \pm 0.15$ dex, in good agreement with Cunha et al. (2007). Their $\alpha$-element abundances are found to be low, following the trends from studies in the outer bulge, and resembling a bar-like population. A refined analysis (Nandakumar et al. 2018) gives a slighter higher mean metallicity of $[\mathrm{Fe} / \mathrm{H}]=+0.3 \pm$ 0.10 dex and confirms the very narrow distribution.

Ryde et al. (2016a) presented an abundance study of $28 \mathrm{M}$ giants in fields located within a few degrees south of the GC using high-resolution $(R \sim 50000)$ spectra. They found a wide range of metallicities that narrows towards the center and confirmed the Rich et al. (2012) study that found alpha enhancement throughout the inner bulge. This would be consistent with a homogeneous enrichment history in the inner bulge.

Very recently, new and more massive samples of chemical abundances from the APOGEE and GIRAFFE Inner Bulge Survey (GIBS) surveys, as well as from high-resolution spectroscopy at Keck and VLT became available. In Sect. 2 we describe this new data set of chemical abundances and in the Sects. 3-5 we will used this dataset to constrain the chemical enrichment of the inner bulge and GC region.

\section{Recent spectroscopy in the inner bulge and in the Galactic Center region}

\subsection{Samples}

Rich et al. (2017) measured metallicities for $17 \mathrm{M}$ giants in the NSC. Membership of those stars is based on detailed extinction measurements as well as on kinematics, meaning proper motions, radial velocities, and orbit calculations. Below, we refer to this sample as R17.

Feldmeier-Krause et al. (2017) used full spectral fitting to derive effective temperatures and metallicities for more than 700 $\mathrm{M}$ giant stars. They found a significant fraction of metal-poor
Table 1. Spectroscopic samples used in this analysis.

\begin{tabular}{cccccc}
\hline \hline Dataset & $(l, b)$ & $N$ & $R$ & $\Delta \lambda(\mu \mathrm{m})$ & Type \\
\hline R17 & $(0,0)$ & 17 & 24000 & $2.11-2.40$ & M giants \\
N18 & $(0,0)$ & 9 & 50000 & $2.08-2.14$ & M giants \\
- & $(0, \pm 1)$ & 23 & - & - & - \\
- & $(0, \pm 2)$ & 24 & - & - & - \\
- & $(0,3)$ & 15 & - & - & - \\
Za19 & $( \pm 0.2, \pm 1)$ & 13 & 22500 & $1.50-1.70$ & M giants \\
- & $( \pm 0.2, \pm 2)$ & 58 & - & - & - \\
- & $( \pm 0.2, \pm 3)$ & 35 & - & - & - \\
Zo17 & $(0,-1)$ & 432 & 6500 & $0.82-0.94$ & RC stars \\
- & $(0,-2)$ & 432 & - & - & - \\
FK17 & $(0,0)$ & 706 & 4000 & $1.93-2.46$ & M giants \\
\hline
\end{tabular}

Notes. The first column gives the reference, the second column the central coordinates in galactic longitudes and latitudes, the third column the number of used stars, the fourth column the spectral resolution, the fifth column the corresponding wavelength range in $\mu m$, and the last column the tracer of the stellar population used.

stars and found also a significant amount of super metal-rich stars with $[\mathrm{Fe} / \mathrm{H}]>0.5$, somewhat ruling out the scenario that the NSC could be entirely formed from the infalling of globular clusters. However, as they mentioned, these extremely metal-rich stars should be regarded with caution. We refer to this sample as FK17.

In the APOGEE DR12 bulge sample García Pérez et al. (2018) identified more than two components in the metallicity distribution by arranging the red giant stars according to their projected Galactocentric distance and distance from the Galactic mid-plane. Fragkoudi et al. (2018) used the metallicities derived from the APOGEE DR13 to compare the observed metallicity distribution function (MDF) with that obtained from N-body simulations of a composite (thin+thick) stellar disk.

Zasowski et al. (2019) presented chemical abundances of M giant stars from the latest APOGEE DR14, in the inner three degrees. We selected stars along the minor axis $\left(l= \pm 0.2^{\circ}\right)$ and at galactic latitudes $\left( \pm 1^{\circ}, \pm 2^{\circ}\right.$, and $\left.\pm 3^{\circ}\right)$. Henceforth, we refer to this sample as Za19.

Zoccali et al. (2017) derived metallicities for 432 stars at $b=-2^{\circ}$ and $b=-1^{\circ}$ from the GIBS survey, using the optical Ca IR triplet method. They found evidence for a bimodal MDF. Using a similar definition of metal rich being suprasolar, and metal poor being subsolar, they argue that the metal rich population is flattened and concentrated toward the plane, while the metal poor component is spheroidal. Henceforth, we refer to this sample as Zo17.

Nandakumar et al. (2018) measured chemical abundances for 71 giant stars in the inner two degrees. We want to emphasize that the stars in the GC field are stars surrounding the NSC and are not members of the NSC. These chemical abundances were obtained by an homogeneous reanalysis of the high-resolution $K$-band spectra of Ryde et al. $(2015,2016 a)$. Surface gravities have been obtained in an iterative way (with respect to $T_{\text {eff }}$ and $[\mathrm{Fe} / \mathrm{H}])$ by placing them on stellar isochrones. The new $K$-band line list (Thorsbro et al. 2018) was also used. Henceforth, we refer to this sample as N18. Table 1 shows the summary of the spectroscopic data set we used in our analysis together with the information about the central coordinates of the samples, the number of stars, the spectral resolution, the wavelength range, as well as the main tracer of the population (e.g., M giants, RC stars). 
Table 2. Derived temperature, gravity, and metallicity for a test giant star common to different studies.

\begin{tabular}{lccc}
\hline \hline & Do et al. (2015) & FK17 & This work \\
\hline$T_{\text {eff }}$ & $3558 \pm 414 \mathrm{~K}$ & $3479 \pm 229 \mathrm{~K}$ & $3200 \pm 200 \mathrm{~K}$ \\
$\log g$ & $3.0 \pm 0.91$ & $0.1 \pm 1.0$ & $0.3 \pm 0.3$ \\
{$[\mathrm{Fe} / \mathrm{H}]$} & $0.80 \pm 0.32$ & $0.3 \pm 0.33$ & $0.2 \pm 0.2$ \\
\hline
\end{tabular}

\subsection{Caveats about chemical abundances from high- and low-resolution spectra}

There is a general debate on whether and eventually how chemical abundances from low-resolution spectra $(R \leq 5000)$ can be compared with those from medium $(R \sim 10000)$ and highresolution $(R \geq 20000)$. In the inner two degrees of the Milky Way that issue is even more critical, given that we are often dealing with metal-rich, cool giants suffering from substantial molecular lines in their spectra, with temperatures well below $4500 \mathrm{~K}$ and $\log g<2$. Appearance of molecular lines and consequent line blending and blanketing, saturation, inaccurate line lists, uncertainties in stellar parameters, etc. can severely affect the derived chemical abundances of $\mathbf{M}$ giants (see e.g., Ryde et al. 2016a; Nandakumar et al. 2018). In addition to all of these problems, some lines are strongly temperature-sensitive due to ionization issues and hyperfine splitting (Thorsbro et al. 2018).

In order to provide a first check of the possible mismatch among stellar parameters obtained form spectra at different resolutions, on 30 July 2017 at Keck II, we obtained a highresolution $(R \sim 23000) K$-band spectrum of the star Nr. 44 from FK17, that is also studied in Do et al. (2015). The star is at $(\mathrm{RA}, \mathrm{Dec})=\left(266.42050^{\circ},-29.006775^{\circ}\right)$ using epoch J2000 and it has $K_{s}=10.4 \mathrm{mag}$. We use the Spectroscopy Made Easy code for spectral synthesis (Valenti \& Piskunov 1996; Valenti 2012) and the MARCS model atmospheres (Gustafsson et al. 2008) in spherical geometry.

Table 2 shows our best-fit stellar parameters and metallicity together with the corresponding values found by Do et al. (2015) and FK17. Our values of the stellar parameters and metallicity for this star are in reasonable agreement with those of FK17 within the uncertainties, while both gravity and $[\mathrm{Fe} / \mathrm{H}]$ by Do et al. (2015) seem improbably large. For this reason, as well as the previously mentioned issue with unphysical gravities and extreme metallicities, we choose to exclude the $R=5000$ sample of Do et al. (2015) from our analysis.

\section{Metallicity distribution function}

By using the state-of-the-art metallicity samples described in Sect. 2, we computed the MDF in the inner two degrees of the Milky Way bulge. Figure 2 shows the individual MDFs of Zo17, Za19, and N18, respectively. Their median metallicity is $-0.04,-0.06$, and 0.04 dex, respectively with a typical rms of $0.4 \mathrm{dex}$, showing that individual MDFs are comparable. In order to maximize the statistical significance of each distribution, we merged the Zo17, N18, and Za19 samples in the inner bulge fields, which is shown in Fig. 3. We refer to this as the "merged sample".

Figure 3 shows also the normalized MDFs for the inner bulge fields at $b= \pm 2^{\circ}$ and $b= \pm 1^{\circ}$ (upper panel), for the GC (middle panel), and for the NSC (bottom panel). The MDF of the inner bulge fields (that is Zo17, N18, and Za19) is very broad, with some evidence of bimodality as is the

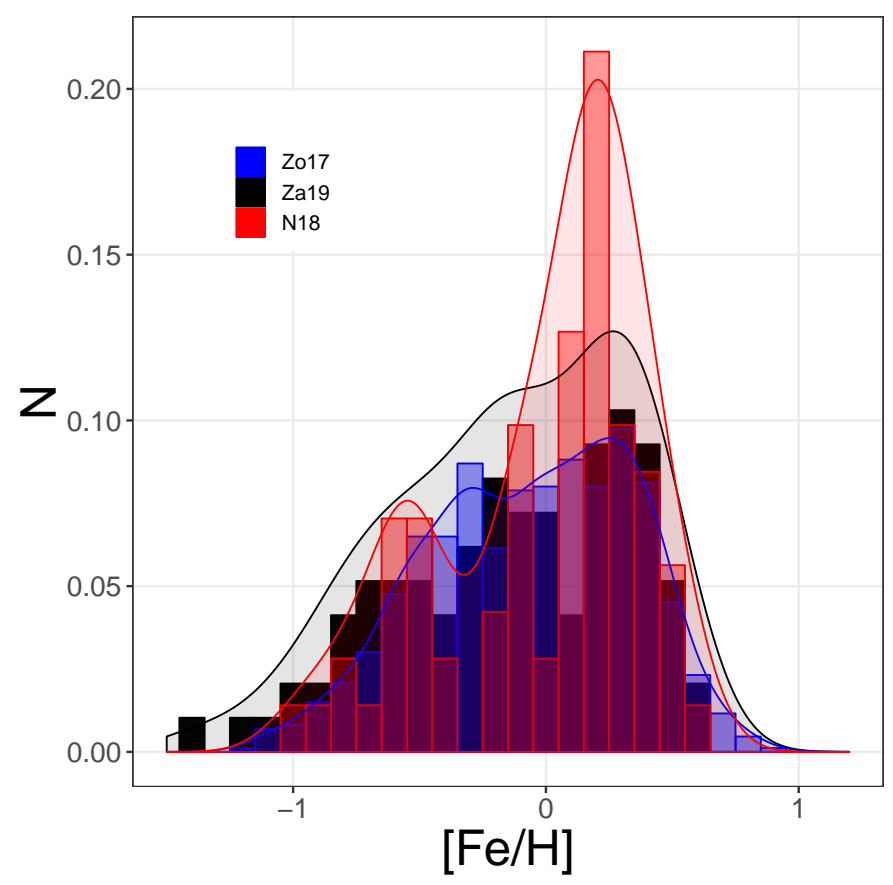

Fig. 2. Normalized Metallicity Distribution Function of $M$ giants between individual samples of Za19 (black), Zo17 (blue), and N18 (red). A kernel density estimation for each of the three samples with a bin size of 0.1 dex is overplotted as a solid line.

case for bulge fields at higher galactic latitudes (e.g., BW). However the two peaks at super-solar and sub-solar metallicity are less pronounced in the inner bulge merged sample. The $\mathrm{MDF}$ in the GC is narrow with a single peak at super-solar metallicity and no metal-poor stars. We want to stress that the GC sample consists of only nine stars and a larger sample is clearly needed before ruling out the presence of metal-poor stars. Future large surveys in the IR such as MOONS at the ESO/VLT (Cirasuolo \& MOONS Consortium 2016) will definitely increase the sample by at least one order of magnitude. The MDF in the NSC is comparable to the GC but broader, with a tail toward subsolar metallicities. In R17 the metal-poor tail is less extended than in FK17 but the sample size of R17 is too small to do a more detailed quantitative analysis. In order to merge the R17 and the FK17 samples, we performed MonteCarlo simulations where from the FK17 sample we randomly extracted 17 stars (which is the sample size of R17) 10000 times. The resulting mean and standard deviation of the extracted samples are within $0.05 \mathrm{dex}$ in $[\mathrm{Fe} / \mathrm{H}]$ of the full $\mathrm{R} 17$ sample. In the following we refer to the GC and the NSC as the GC region.

The most striking difference between the MDF in the inner bulge and in the GC region is the larger population of metalpoor stars in the former. This difference is significant. Indeed, a statistical KS-test gives a $p$-value of 0.02 , indicating that these two distributions do not belong to the same population. Such a difference would favor a scenario of an in-situ formation of the NSC. However, infall of complex stellar systems such as Terzan 5 (Origlia et al. 2013, 2019) with major age and metallicity spreads or genuine globular clusters (Capuzzo-Dolcetta \& Miocchi 2008; Tsatsi et al. 2017) cannot be fully ruled out as possible contributors. In the latter case, chemical footprints of dispersion in light elements (Schiavon et al. 2017) should be detected, and chemical abundances for these metals are urgently needed. 

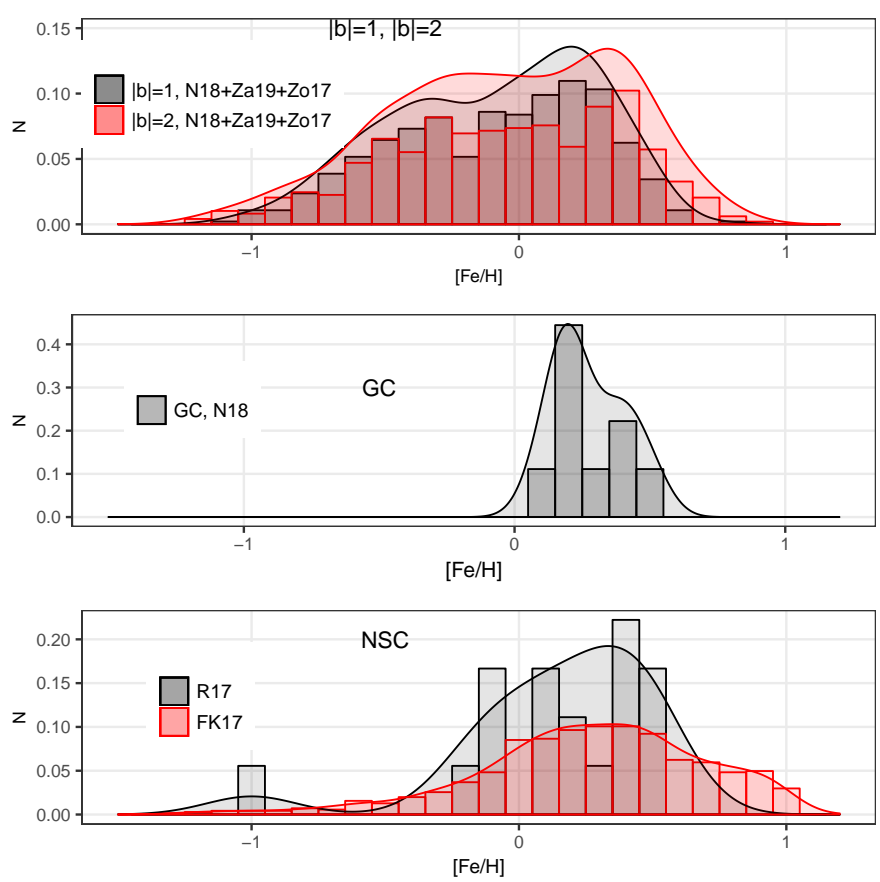

Fig. 3. Normalized Metallicity Distribution Function of $M$ giants in inner two degrees. Upper panel: distributions at $|b|=1 \mathrm{deg}$ and $|b|=$ 2 deg from Zo17, N18, and Za19. Middle panel: same for the GC field from N18, and lower panel: for the NSC from FK17 and R17. A kernel density estimation with a bin size of $0.1 \mathrm{dex}$ is also overplotted as a solid line.

\section{Metallicity gradients}

In order to consistently trace possible vertical and radial gradients in the Galactic bulge, it is important to quantify the relative contribution of the supersolar, metal-rich (hereafter MR) and the subsolar, metal-poor (hearafter MP) stars. Indeed, when the MDFs are complex as in the Galactic bulge, a straightforward average value is not always appropriate to describe the metal content of the sampled stellar population at a given distance from the GC.

We thus divided each sample described in Sect. 2 into two components: the MR with $[\mathrm{Fe} / \mathrm{H}]>0$ and the MP with $[\mathrm{Fe} / \mathrm{H}]<0$, following Zoccali et al. (2017). Then we computed the number ratio of MR to MP stars and their average metallicities. We verified that slightly different (by \pm 0.2 dex from $[\mathrm{Fe} / \mathrm{H}]=0.0$ ) assumptions to separate MR and MP stars do not significantly affect the results. In this respect, Nandakumar et al. (2017) already demonstrated that the effect of the selection function plays a minor role in MDF studies.

\subsection{The vertical metallicity gradient}

By using the same state-of-the-art metallicity samples as for the MDFs, we derived number ratios of MR to MP stars and their average metallicities, and we investigate their behavior as a function of the distance $(z)$ from the Galactic plane. Here for the merged sample at $z=0$ we used the data set of R17, FK17, and the stars at the GC from N18. For the merged sample of $|b|=1^{\circ},|b|=2^{\circ}$, and $|b|=3^{\circ}$, we used the corresponding data sets of N18, Za18, and Zo17 as described in Sect. 3. Figure 4 shows the fraction of MR to MP stars with varying $z$. For comparison we also included the corresponding values at $b= \pm 3$ deg from Nandakumar et al. (2018) and Zasowski et al. (2019), and

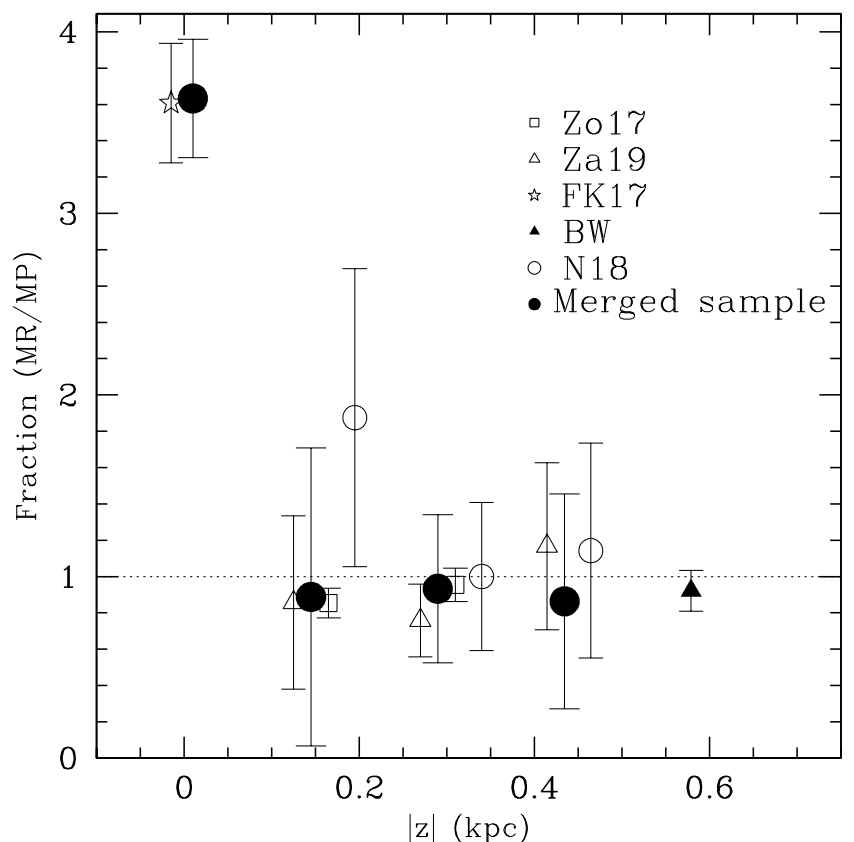

Fig. 4. Fraction of $\mathrm{MR}([\mathrm{Fe} / \mathrm{H}]>0)$ to $\mathrm{MP}$ stars $([\mathrm{Fe} / \mathrm{H}<0)$ as a function of vertical distance for considered samples of stars. Errorbars have been computed as $\Delta f=f \times \sqrt{(1+f) / \mathrm{MR}}$, where $f$ is the number ratio of MR to MP stars and MR is the number of MR stars.

in BW from Schultheis et al. (2017). The fraction of MR to MP stars for $|b| \geq 1^{\circ}$ (corresponding to $z=0.14 \mathrm{kpc}$ ) out to the BW at $z=0.57 \mathrm{kpc}$ turns out to be rather constant around the value of 1 , suggesting an almost equal fraction of MR and MP stars (see also Zoccali et al. 2017). At variance, in the GC region the MR/MP number ratio significantly increases up to a value of about 3.6, indicating a dominance of the MR component. Figure 5 shows the map of the MR/MP number ratio in the innermost bulge region while in Table 3 we list the fraction of MR/MP stars for each field. For comparison we also show the ratio of MR to MP stars from Zoccali et al. (2018) based on the GIBS data (see their Table 1 ) but confined to $|b| \leq 3^{\circ}$. The distribution is rather flat, with values ranging between 0.8 and 1.2 with a spike in the GC region. In Fig. 5, we superimpose the stellar mass distribution of the nuclear disk from Launhardt et al. (2002) where our metallicity peak corresponds with the concentration of the mass within the nuclear disk/NSC. Subsequent studies all confirm the extreme concentration of stellar mass in the nuclear disk and the NSC (e.g., Fritz et al. 2016). Interestingly enough, the individual samples and the merged ones (i.e., FK17+R17 in the GC region, $\mathrm{N} 18+\mathrm{Za} 19+\mathrm{Zo} 17$ in the $b= \pm 1$ and $b=$ \pm 2 fields, and N18+Za19 in the $b= \pm 3$ field) show similar MR/MP number ratios, despite their different spectral resolution, line diagnostics, and statistical significance. This suggests that the merged samples, meaning those with the highest statistical significance, can be safely used to trace possible metallicity variations. Our finding that different spectral resolution and line diagnostics provide similar results is not surprising, given that we are simply considering two broad metallicity categories (i.e., MR and MP).

Figure 6 shows the median metallicities for the individual MR and MP components as well as for the global metallicity, as a function of $z$. We only used the merged samples since they are statistically more significant and we performed a linear, square fit to the data points. The coefficients are reported in Table 4. Very small positive slopes in the inner bulge fields (thus 


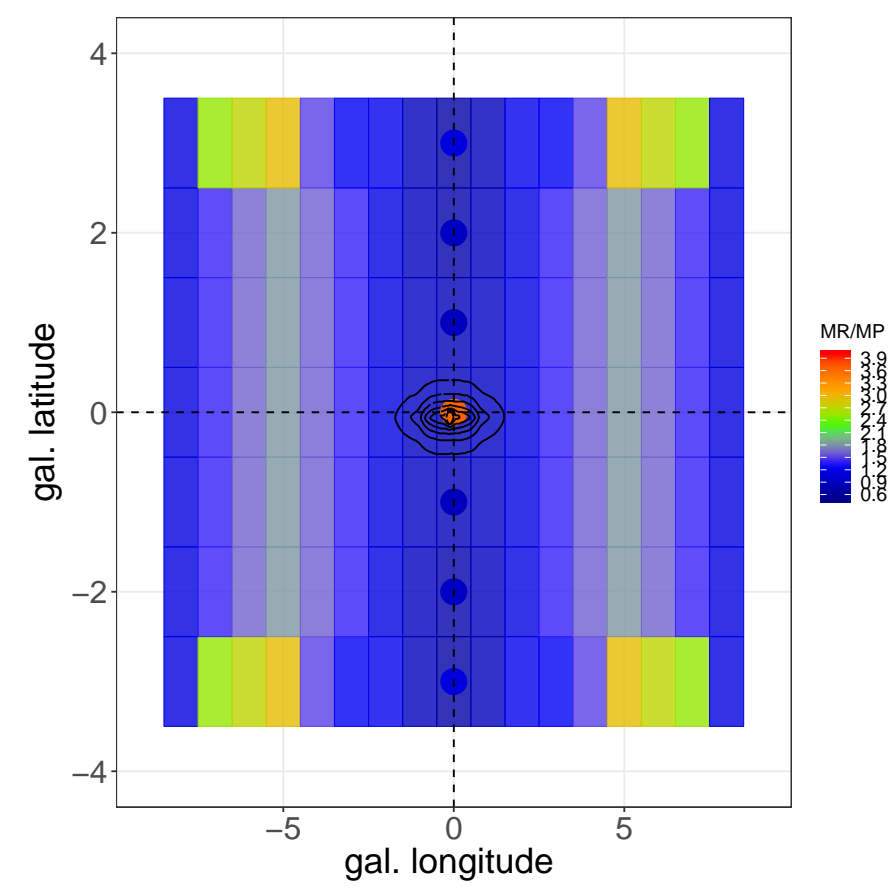

Fig. 5. Fraction of metal-rich to metal-poor stars (filled circles) superimposed by fraction of MR to MP stars from Zoccali et al. (2018, their Table 1). The black contours show the surface brightness map of the best-fit model of the "nuclear bulge" component by Launhardt et al. (2002).

Table 3. Ratio of MR to MP stars for each spectroscopic sample and each merge sample.

\begin{tabular}{llll}
\hline \hline Data set & $(l, b)$ & Ratio $(\mathrm{MR} / \mathrm{MP})$ & $\sigma_{(\mathrm{MR} / \mathrm{MP})}$ \\
\hline FK17 & $(0,0)$ & 3.61 & 0.30 \\
FK17+R17+N18 & $(0,0)$ & 3.63 & 0.33 \\
N18 & $(0, \pm 1)$ & 1.87 & 0.82 \\
N18 & $(0, \pm 2)$ & 1.00 & 0.41 \\
N18 & $(0,3)$ & 1.14 & 0.59 \\
Zo17 & $(0,-1)$ & 0.85 & 0.08 \\
Zo17 & $(0,-2)$ & 0.95 & 0.09 \\
Za19 & $( \pm 0.2, \pm 1)$ & 0.86 & 0.48 \\
Za19 & $( \pm 0.2, \pm 2)$ & 0.76 & 0.20 \\
Za19 & $( \pm 0.2, \pm 3)$ & 1.17 & 0.46 \\
N18+Zo17+Za19 & $(0, \pm 1)$ & 0.89 & 0.82 \\
N18+Zo17+Za19 & $(0, \pm 2)$ & 0.93 & 0.41 \\
N18+Za19 & $(0, \pm 3)$ & 0.86 & 0.59 \\
BW & $(1,-4)$ & 0.92 & 0.11 \\
\hline
\end{tabular}

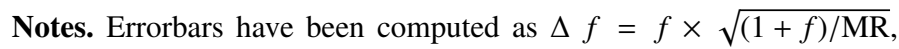
where $f$ is the number ratio of MR to MP stars and MR is the number of MR stars.

excluding the GC region) are obtained, the highest value for the MP component, indicating that the mild (if any) positive gradient is mostly due to the decrease of the median metallicity of the MP component moving outwards. At variance, if the GC region is included, slopes become negative and the mild negative gradients are practically due to the high median metallicity of the dominant MR population in the GC region. Interestingly, all the slopes in Table 4 are consistent with flat distributions at $\leq 1.5 \sigma$ level.

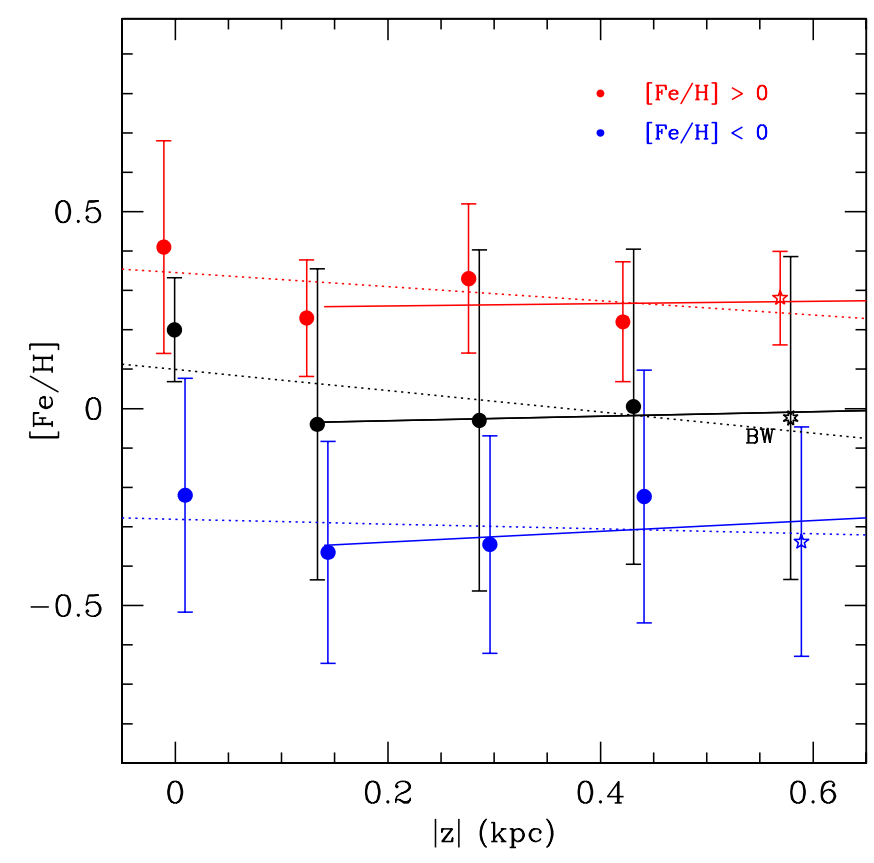

Fig. 6. Vertical metallicity gradient in inner bulge and GC region. Shown are results for MR (red dots), MP (blue dots), and all the stars together (black dots) from the merged samples of FK17+R17 in the GC region, $\mathrm{N} 18+\mathrm{Za} 19+\mathrm{Zo} 17$ in the $b= \pm 1^{\circ}$ and $b= \pm 2^{\circ}$ fields, $\mathrm{N} 18+\mathrm{Za} 19$ in the $b= \pm 3^{\circ}$ field, and results from Schultheis et al. (2017) in the BW. Errorbars refer to $1 \sigma$ dispersions. Dotted lines show the linear fit including the GC region, while the solid lines show the fit omitting the $\mathrm{GC}$ region.

Table 4. Linear fitting (i.e., $y=a 1 * x+a 0$ ) parameters and $1 \sigma$ dispersions for vertical metallicity gradient.

\begin{tabular}{ccccccc}
\hline \hline Fit & All & AllB & MRall & MPall & MRB & MPB \\
\hline & & & & & & \\
$\mathrm{a} 0$ & 0.099 & -0.042 & 0.345 & -0.281 & 0.254 & -0.367 \\
$\sigma_{a 0}$ & 0.069 & 0.023 & 0.059 & 0.061 & 0.073 & 0.085 \\
$\mathrm{a} 1$ & -0.270 & 0.057 & -0.179 & -0.061 & 0.031 & 0.136 \\
$\sigma_{\mathrm{a} 1}$ & 0.198 & 0.058 & 0.165 & 0.174 & 0.182 & 0.212 \\
$\mathrm{rms}$ & 0.09 & 0.03 & 0.08 & 0.07 & 0.06 & 0.07 \\
\hline
\end{tabular}

Notes. The overall $\mathrm{rms}$ of the residuals is given in the last row. The second column (All) shows the parameters for the full sample, the third one (AllB) for the full sample in the inner bulge without the GC, the fourth and fifth ones for the MR and the MP sub-samples including the $\mathrm{GC}$, and columns six and seven for the MR and MP populations in the inner bulge without the GC.

\subsection{The radial metallicity gradient}

The radial metallicity gradient is an important constraint on the chemical evolution for the Milky Way and external galaxies. Figure 7 shows the mean metallicity for open clusters and Cepheids in the range $5<R_{\mathrm{GC}}<15 \mathrm{kpc}$ by Genovali et al. (2014) and Netopil et al. (2016) as a function of the Galactocentric distance and the fitting relations based on observations of field giant stars by Hayden et al. (2014) and Anders et al. (2017). For comparison, mean metallicities for the GC region and the inner bulge fields as discussed in this paper are also reported. There is clear evidence that the extrapolation of the radial gradient inferred at large Galactocentric distances to the center would result in a metallicity exceeding the measured central value by 


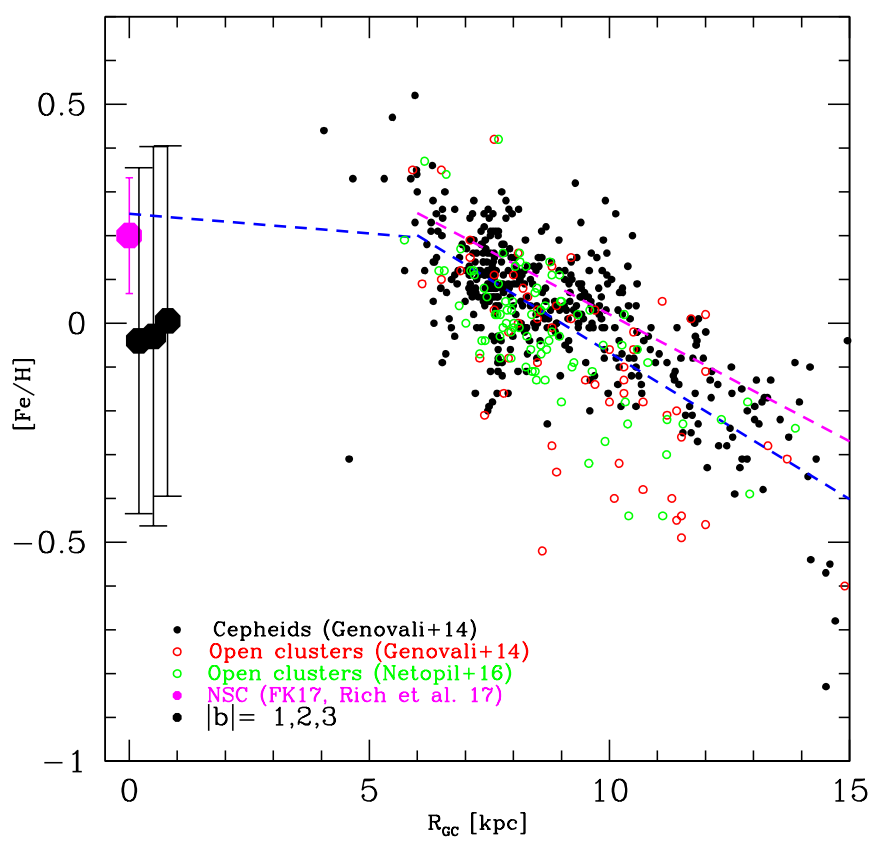

Fig. 7. Radial gradient close to Galactic plane. Shown are data as traced by Cepheids (black dots from Genovali et al. 2014), open clusters (red circles from Genovali et al. 2014 and green circles from Netopil et al. 2016), and big magenta and black dots are the average metallicities for the GC region and $|b|=1,2,3$ deg inner bulge fields discussed in this work. Errorbars refer to $1 \sigma$ dispersions. The blue, dashed line indicates the relation noted by Hayden et al. (2014) for a large sample of APOGEE $M$ giant stars covering a wide range of Galactocentric distances. The magenta dashed line indicates the relation found by Anders et al. (2017) for red giant stars with well-determined asteroseismological parameters.

$\sim 0.6$ dex. All the measurements in the inner bulge and in the $\mathrm{GC}$ region indicate average metallicities between solar and twice solar at most. As already speculated by Hayden et al. (2014), this flattening might arise due to the mixing of the stars in the presence of the galactic bar.

\section{Alpha-element abundances}

In order to determine individual abundances, high-spectral resolution $(R>20000)$ is necessary. Indeed, very few studies exist in the inner two degrees on detailed chemical abundances of alphaelements in the bulge low-latitude fields. Rich et al. (2007) did the first detailed chemical abundance analysis and found a rather homogeneous $\alpha$-enhancement of $\sim 0.3$ dex up to solar metallicity for a sample of $17 \mathrm{M}$ giants located at $(l, b)=(0,-1)$. In a similar study Rich et al. (2012) extended this work to two more fields finding the same average $\alpha$-element enhancement of $\sim 0.3$ dex. Also, the study of the metal-poor stars in the NSC $([\mathrm{Fe} / \mathrm{H}] \sim$ -1 ) found by Ryde et al. (2016b), shows an $\alpha$-enhancement of $\sim 0.4$ dex.

Figure 8 shows the $[\mathrm{Mg} / \mathrm{Fe}]$ and $[\mathrm{Si} / \mathrm{Fe}]$ abundance ratios as a function of $[\mathrm{Fe} / \mathrm{H}]$ as obtained by Nandakumar et al. (2018) in the central two degrees and by Zasowski et al. (2019) using the latest APOGEE Data Release 14 of bulge chemical abundances. However, we see that the APOGEE $[\mathrm{Mg} / \mathrm{Fe}]$ and $[\mathrm{Si} / \mathrm{Fe}]$ abundances level off approximately above solar metallicity, while our $\alpha$-abundances continue decreasing with increasing metallicity. Matteucci et al. (2019) reproduces this flattening by artificially increasing the $\mathrm{Mg}$ produced by Type Ia supernovae by a factor of ten. However, we believe that this flattening is unphysical, and results from an artifact in the analysis of metal-rich, cool M giants.

In the $|b|=1^{\circ}$ and $|b|=2^{\circ}$ fields we observe a general decline of the $[\mathrm{Mg} / \mathrm{Fe}]$ abundance ratio with increasing metallicity down to roughly solar-scaled $[\mathrm{Mg} / \mathrm{Fe}]$ at super-solar $[\mathrm{Fe} / \mathrm{H}]$, similar to that found by APOGEE, though with larger scatter. The $[\mathrm{Si} / \mathrm{Fe}]$ distributions tend to be systematically lower (by $0.1-0.2 \mathrm{dex}$ ) than APOGEE, especially at $[\mathrm{Fe} / \mathrm{H}]>-0.5$ dex. The $\mathrm{GC}$ distributions follow those of the $|b|=1^{\circ}$ and $|b|=2^{\circ}$ fields at supersolar $[\mathrm{Fe} / \mathrm{H}]$. However, we want to stress that the GC sample is very small and clearly more observations are necessary for a more quantitative comparison.

\section{Discussion and conclusions}

Based on high- and low-resolution spectroscopic observations of giant stars located in the inner two degrees of the galactic bulge, we find evidence of a dominant population of metal-rich stars in the GC region, leading to an average metallicity of +0.2 dex. Moving outward from the GC, in the central $600 \mathrm{pc}$ we find that the ratio of meta-rich to metal-poor stars is constant, with no evidence of vertical abundance gradients, confirming on a more robust statistical basis the previous work of Ramírez et al. (2000b), Rich et al. (2007, 2012), and Babusiaux et al. (2014).

The GC region hosts a complex stellar population with a large spread in age and chemical content that significantly differs from the bulge stellar population in the inner $600 \mathrm{pc}$. Hence, in order to properly assess the possible presence of vertical or radial gradients in the bulge and inner disk, the GC region should be excluded. Interestingly, the vertical metallicity gradient of about $-0.3 \mathrm{dex} \mathrm{kpc}^{-1}$ (Rojas-Arriagada et al. 2017; Gonzalez et al. 2013) measured at galactic latitudes $b \geq 4^{\circ}$ also holds in the inner $600 \mathrm{pc}$ though only if the GC region is included; a proper measurement of the gradient should not consider the GC region.

We can thus confidently state that the metallicity distribution in the inner $600 \mathrm{pc}$ of the Galactic bulge is rather homogeneous. Only the innermost $\sim 10 \mathrm{pc}$ shows an overall higher mean metallicity, suggesting that the GC region is a very peculiar environment with a large concentration of MR stars, perhaps arising from the retention of metal-rich gas by the potential well of the NSC. It is worth noting that also the radial metallicity gradient observed in the disk at Galactocentric distances $>6 \mathrm{kpc}$ does not extrapolate when moving inwards toward the low-latitude bulge fields and the GC region.

There have been various mechanisms proposed to explain the origin of the vertical metallicity gradient of the MW bulge. It was originally thought that a dispersion-dominated classical bulge/spheroid was the only possible explanation for the vertical metallicity gradient (e.g. Zoccali et al. 2008). However, other studies have shown that the redistribution of stars by a bar and a boxy peanut bulge can also produce a vertical metallicity gradient (see for example Martinez-Valpuesta \& Gerhard 2013; Di Matteo et al. 2014). Fragkoudi et al. (2017) showed that while such models can indeed reproduce the vertical metallicity gradient, they do not reproduce a number of other trends seen in the MW bulge and inner disk, such as the metal-poor stars in innermost regions and the positive longitudinal gradient.

Very few chemical evolution models exist in the inner two degrees. Grieco et al. (2015) studied the chemical evolution of the central region and concluded that the stars in the GC formed very rapidly $(0.1-0.7 \mathrm{Gyr})$ with high star formation efficiency $\left(25 \mathrm{Gyr}^{-1}\right)$ and a top-heavy initial mass function. Fragkoudi et al. (2018) used a composite model of a thin and a 

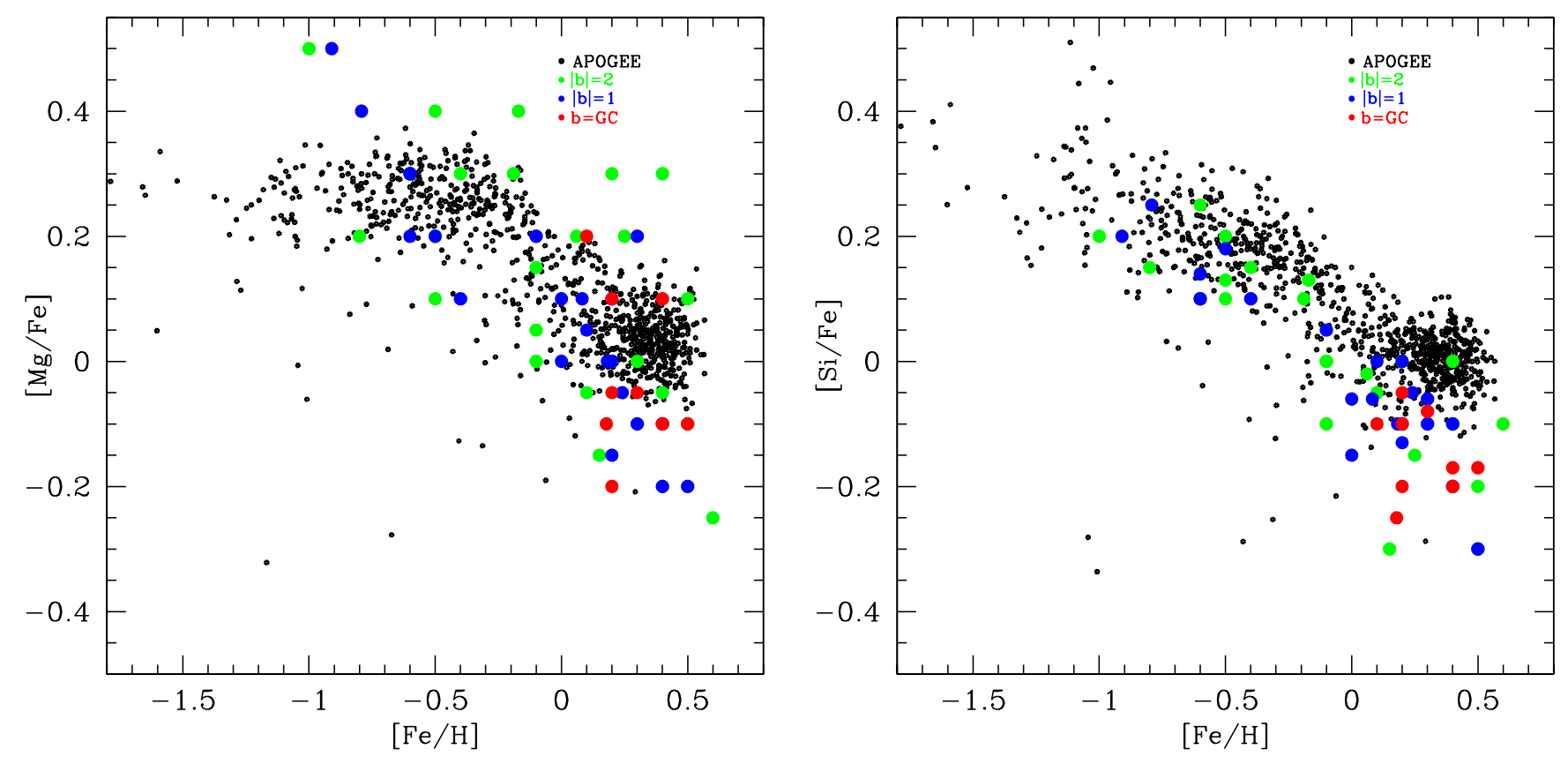

Fig. 8. $[\mathrm{Mg} / \mathrm{Fe}]$ vs. $[\mathrm{Fe} / \mathrm{H}]$ (left panel) and $[\mathrm{Si} / \mathrm{Fe}]$ vs. $[\mathrm{Fe} / \mathrm{H}]$ (right panel) trends in inner bulge and $\mathrm{GC}$ regions.

massive centrally concentrated thick disk that evolves in forming a bar and a boxy/peanut bulge to find a remarkably good agreement with the metallicity distribution functions of APOGEE. They predict a gradient of $-0.10 \mathrm{dex} \mathrm{kpc}^{-1}$ for the inner $500 \mathrm{pc}$. However, this model is a simple, closed-box model that does not include star formation and stellar feedback processes nor any gaseous physics. The presence of RR Lyrae stars in the NSC (Dong et al. 2017) favors at least a non-negligible portion of the population being $\sim 10 \mathrm{Gyr}$ or older, a point that is also made by Nogueras-Lara et al. (2018b).

Our evidence for a high metallicity peak in the GC might plausibly be explained by a rapid in-situ star formation event in an enriched gas environment, which might have involved coevolution of the super massive black hole growth and the NSC. The formation of multiple stellar populations in the NSC might well be problematic due to the unique environment of $\operatorname{SgrA}^{*}$ (e.g., a strong tidal field from the $\mathrm{SMBH}$ ) and also at some point in its history would have included substantial AGN activity. Further, high energy phenomena are observed at the present day, including magnetic fields and outflows of hot gas (Ponti et al. 2019). It is possible that the conditions that produced the supermassive black hole arose at the same time vigorous star formation was building the NSC, leaving the fossil remnant of high metallicity that we observe today. However, the strong tidal fields near Sgr $\mathrm{A}^{*}$ remain as an unsolved challenge to explaining star formation, also known as the so-called paradox of youth (Ghez et al. 2003). An attractive idea is to attribute the observed high metallicity spike to enhanced star formation rates in AGN hosts compared to non-AGN that appear to be the case (Santini et al. 2012) so they appear more pronounced for high luminosity AGNs. On the other hand, Stanley et al. (2015) found no evidence of a correlation between star formation rate and AGN activity across all AGN luminosities.

It would be valuable to test this hypothesis by measuring the star formation history and metallicity of other NSCs, perhaps spanning a range in black hole mass. It is also important to use the next generation of facilities to constrain in greater detail the star formation of the NSC and surrounding nuclear disk and bulge population.
We have exploited the high luminosities of late $\mathrm{M}$ giants to permit the investigation of the NSC using the current generation of instrumentation. Spectrographs coming online in the near future, such as CRIRES+ or MOONS at the VLT, and, later on HIRES, HARMONI, and MOSAIC at the ELT, will allow a deeper exploration of this region that may eventually include the red clump stars. The NSC offers our best opportunity to explore the coevolution of a stellar population and a supermassive black hole; such studies will be of great interest in the next decade.

Acknowledgements. We want to thank the referee E. Valenti for her very constructive comments. We want to thank F. Fragkoudi and A. Mastrobueno-Battisti for very fruitful discussions. M.S. acknowledges the Programme National de Cosmologie et Galaxies (PNCG) of CNRS/INSU, France, for financial support. N.R. acknowledge support from the Swedish Research Council, VR (project number 621-2014-5640), funds from Kungl. Fysiografiska Sällskapet i Lund. (Stiftelsen Walter Gyllenbergs fond and Märta och Erik Holmbergs donation), the project grant "The New Milky" from the Knut and Alice Wallenberg foundation, and support from the Crafoord Foundation, and Stiftelsen Olle Engkvist Byggmästare. Some of the data presented herein were obtained at the W. M. Keck Observatory, which is operated as a scientific partnership among the California Institute of Technology, the University of California, and the National Aeronautics and Space Administration. The Observatory was made possible by the generous financial support of the W. M. Keck Foundation. The authors wish to recognize and acknowledge the very significant cultural role and reverence that the summit of Mauna Kea has always had within the indigenous Hawaiian community. We are most fortunate to have the opportunity to conduct observations from this mountain.

\section{References}

Anders, F., Chiappini, C., Minchev, I., et al. 2017, A\&A, 600, A70 Antonini, F. 2013, ApJ, 763, 62

Babusiaux, C., Katz, D., Hill, V., et al. 2014, A\&A, 563, A15

Barbuy, B., Chiappini, C., \& Gerhard, O. 2018, ARA\&A, 56, 223

Capuzzo-Dolcetta, R. 1993, ApJ, 415, 616

Capuzzo-Dolcetta, R., \& Miocchi, P. 2008, ApJ, 681, 1136

Carr, J. S., Sellgren, K., \& Balachandran, S. C. 2000, ApJ, 530, 307

Cirasuolo, M. \& MOONS Consortium 2016, in Multi-Object Spectroscopy in the

Next Decade: Big Questions, Large Surveys, and Wide Fields, eds. I. Skillen,

M. Balcells, \& S. Trager, ASP Conf. Ser., 507, 109

Cunha, K., \& Smith, V. V. 2006, ApJ, 651, 491

Cunha, K., Sellgren, K., Smith, V. V., et al. 2007, ApJ, 669, 1011 
Di Matteo, P., Haywood, M., Gómez, A., et al. 2014, A\&A, 567, A122 Do, T., Kerzendorf, W., Winsor, N., et al. 2015, ApJ, 809, 143

Dong, H., Schödel, R., Williams, B. F., et al. 2017, MNRAS, 471, 3617

Feldmeier, A., Neumayer, N., Seth, A., et al. 2014, A\&A, 570, A2

Feldmeier-Krause, A., Kerzendorf, W., Neumayer, N., et al. 2017, MNRAS, 464, 194

Fragkoudi, F., Di Matteo, P., Haywood, M., et al. 2017, A\&A, 606, A47

Fragkoudi, F., Di Matteo, P., Haywood, M., et al. 2018, A\&A, 616, A180

Fritz, T. K., Gillessen, S., Dodds-Eden, K., et al. 2011, ApJ, 737, 73

Fritz, T. K., Chatzopoulos, S., Gerhard, O., et al. 2016, ApJ, 821, 44

García Pérez, A. E., Ness, M., Robin, A. C., et al. 2018, ApJ, 852, 91

Genovali, K., Lemasle, B., Bono, G., et al. 2014, A\&A, 566, A37

Genzel, R., Eisenhauer, F., \& Gillessen, S. 2010, Rev. Mod. Phys., 82, 3121

Ghez, A. M., Duchêne, G., Matthews, K., et al. 2003, ApJ, 586, L127

Gnedin, O. Y., Ostriker, J. P., \& Tremaine, S. 2014, ApJ, 785, 71

Gonzalez, O. A., Rejkuba, M., Zoccali, M., et al. 2012, A\&A, 543, A13

Gonzalez, O. A., Rejkuba, M., Zoccali, M., et al. 2013, A\&A, 552, A110

Grieco, V., Matteucci, F., Ryde, N., Schultheis, M., \& Uttenthaler, S. 2015 MNRAS, 450, 2094

Gustafsson, B., Edvardsson, B., Eriksson, K., et al. 2008, A\&A, 486, 951

Hayden, M. R., Holtzman, J. A., Bovy, J., et al. 2014, AJ, 147, 116

Karim, M. T., \& Mamajek, E. E. 2017, MNRAS, 465, 472

Launhardt, R., Zylka, R., \& Mezger, P. G. 2002, A\&A, 384, 112

Majewski, S. R., Schiavon, R. P., Frinchaboy, P. M., et al. 2017, AJ, 154, 94

Martinez-Valpuesta, I., \& Gerhard, O. 2013, ApJ, 766, L3

Matteucci, F., Grisoni, V., Spitoni, E., et al. 2019, MNRAS, 487, 5363

McLean, I. S. 2005, in High Resolution Infrared Spectroscopy in Astronomy, eds. H. U. Käufl, R. Siebenmorgen, \& A. F. M. Moorwood, 25

Milosavljević, M. 2004, ApJ, 605, L13

Nandakumar, G., Schultheis, M., Hayden, M., et al. 2017, A\&A, 606, A97

Nandakumar, G., Ryde, N., Schultheis, M., et al. 2018, MNRAS, 478, 4374

Netopil, M., Paunzen, E., Heiter, U., \& Soubiran, C. 2016, A\&A, 585, A150

Neumayer, N. 2017, in Formation, Evolution, and Survival of Massive Star Clusters, eds. C. Charbonnel, \& A. Nota, IAU Symp., 316, 84

Nogueras-Lara, F., Gallego-Calvente, A. T., Dong, H., et al. 2018a, A\&A, 610, A83

Nogueras-Lara, F., Schödel, R., Dong, H., et al. 2018b, A\&A, 620, A83

Origlia, L. 2014, in Setting the scene for Gaia and LAMOST, eds. S. Feltzing, G. Zhao, N. A. Walton, \& P. Whitelock, IAU Symp., 298, 28

Origlia, L., Massari, D., Rich, R. M., et al. 2013, ApJ, 779, L5

Origlia, L., Mucciarelli, A., Fiorentino, G., et al. 2019, ApJ, 871, 114
Pflamm-Altenburg, J., \& Kroupa, P. 2009, MNRAS, 397, 488

Ponti, G., Hofmann, F., Churazov, E., et al. 2019, Nature, 567, 347

Ramírez, S. V., Sellgren, K., Carr, J. S., et al. 2000a, ApJ, 537, 205

Ramírez, S. V., Stephens, A. W., Frogel, J. A., \& DePoy, D. L. 2000b, AJ, 120, 833

Rich, R. M. 2013, in Planets, Stars and Stellar Systems. Volume 5: Galactic Structure and Stellar Populations, eds. T. D. Oswalt, \& G. Gilmore (Netherlands: Springer), 271

Rich, R. M., \& Origlia, L. 2005, ApJ, 634, 1293

Rich, R. M., Origlia, L., \& Valenti, E. 2007, ApJ, 665, L119

Rich, R. M., Origlia, L., \& Valenti, E. 2012, ApJ, 746, 59

Rich, R. M., Ryde, N., Thorsbro, B., et al. 2017, AJ, 154, 239

Rojas-Arriagada, A., Recio-Blanco, A., de Laverny, P., et al. 2017, A\&A, 601, A 140

Ryde, N., \& Schultheis, M. 2015, A\&A, 573, A14

Ryde, N., Lambert, J., Farzone, M., et al. 2015, A\&A, 573, A28

Ryde, N., Schultheis, M., Grieco, V., et al. 2016a, AJ, 151, 1

Ryde, N., Fritz, T. K., Rich, R. M., et al. 2016b, ApJ, 831, 40

Santini, P., Rosario, D. J., Shao, L., et al. 2012, A\&A, 540, A109

Schiavon, R. P., Johnson, J. A., Frinchaboy, P. M., et al. 2017, MNRAS, 466 1010

Schödel, R., Feldmeier, A., Kunneriath, D., et al. 2014, A\&A, 566, A47

Schultheis, M., Sellgren, K., Ramírez, S., et al. 2009, A\&A, 495, 157

Schultheis, M., Chen, B. Q., Jiang, B. W., et al. 2014, A\&A, 566, A120

Schultheis, M., Cunha, K., Zasowski, G., et al. 2015, A\&A, 584, A45

Schultheis, M., Rojas-Arriagada, A., García Pérez, A. E., et al. 2017, A\&A, 600, A14

Seth, A. C., Blum, R. D., Bastian, N., Caldwell, N., \& Debattista, V. P. 2008, ApJ, 687, 997

Stanley, F., Harrison, C. M., Alexander, D. M., et al. 2015, MNRAS, 453, 591

Thorsbro, B., Ryde, N., Schultheis, M., et al. 2018, ApJ, 866, 52

Trapp, A. C., Rich, R. M., Morris, M. R., et al. 2018, ApJ, 861, 75

Tremaine, S. D., Ostriker, J. P., \& Spitzer, Jr., L. 1975, ApJ, 196, 407

Tsatsi, A., Mastrobuono-Battisti, A., van de Ven, G., et al. 2017, MNRAS, 464, 3720

Valenti, J. A., \& Piskunov, N. 1996, A\&AS, 118, 595

Valenti, J. A. 2012, Astrophysics Source Code Library [record ascl:1202 .013]

Zasowski, G., Schultheis, M., Hasselquist, S., et al. 2019, ApJ, 870, 138

Zoccali, M., Hill, V., Lecureur, A., et al. 2008, A\&A, 486, 177

Zoccali, M., Vasquez, S., Gonzalez, O. A., et al. 2017, A\&A, 599, A12

Zoccali, M., Valenti, E., \& Gonzalez, O. A. 2018, A\&A, 618, A147 